\title{
Difficulties for extending Wegner and colleagues' model of the sense of agency to deficits in delusions of alien control
}

\section{Glenn Carruthers}

ARC Centre of Excellence in Cognition and its Disorders

Macquarie University

Glenn.rj.carruthers[]gmail.com

Received November 2012; accepted January 2014; published winter 2014/2015.

\begin{abstract}
Wegner and colleagues have offered an explanation of the sense of agency over one's bodily actions. If the orthodox view is correct and there is a sense of agency deficit associated with delusions of alien control, then Wegner and colleagues' model ought to extend to an explanation of this deficit. Data from intentional binding studies opens up the possibility that an abnormality in representing the timing of mental events leads to a violation of the principle of priority in those suffering from delusions of alien control. However, the model ultimately fails as it is unable to accommodate action monitoring deficits associated with delusions of alien control, in particular the theory of mind deficiencies predicted by such accounts have not materialised. There are theory of mind problems associated with schizophrenia but they are not associated with delusions of alien control. Such accounts thereby fail to adequately explain delusions of alien control.
\end{abstract}

Keywords: sense of agency; self-consciousness; Wegner; delusions; delusions of alien control.

\section{Introduction}

In this paper I argue the model of the sense of agency advocated by Wegner and colleagues is unlikely to be extendable to account for sense of agency deficits in delusions of alien control. This delusion seems to involve a deficit in the sense of being the agent of bodily actions, a sense which functions to help us tell the effects of our actions on the world from the effects of others and non-agents. The sense of agency thus ought to, and is typically conceptualised as, having a tight link to action monitoring. After presenting Wegner and colleagues' model I argue that if those suffering from delusions of alien control 
represent their actions as occurring later than they should, then the link between intentional binding abnormalities, a lack of sensory attenuation for self-produced stimuli and sense of agency deficits observed in delusions of alien control can be explained. Such an abnormality could cause a sense of agency deficit on this model as well as other abnormalities. However, this does not seem applicable to the action monitoring problems associated with delusions of alien control. Further, there seems to be no other way to explain the link between sense of agency deficits and action monitoring problems in Wegner and colleagues' model. Whilst there are hypotheses which could explain this link they are either false or, at best, not supported.

\section{Delusions of Alien Control as a Sense of Agency Deficit}

One key abnormality in the unusual experiences of those suffering from delusions of alien control can be characterised as an unusual sense of agency. Specifically, patients suffering from this disorder report that others seem to be performing actions using their body. Here are some common reports that are often put forward as typical examples of the delusion:

When I reach my hand for the comb it is my hand and arm which move, and my fingers pick up the pen, but I don't control them... I sit there watching them move, and they are quite independent, what they do is nothing to do with me... I am just a puppet who is manipulated by cosmic strings. When the strings are pulled my body moves and I cannot prevent it (Mellor 1970: 18).

I felt like an automaton, guided by a female spirit who had entered me during it [an arm movement] (Spence 2002: 165).

I thought you [the experimenter] were varying the movements with your thoughts (Spence 2002: 165).

I could feel God guiding me [during an arm movement] (Spence 2002:165)

What we see in these reports is the patient attributing the agency behind their action (or perhaps better, the action performed by their body) to another agent. These patients know full well that it is their body which is moving to perform action, but they deny that they are the agent of the action (Gallagher 2000). There is no necessary relationship between this delusion and other delusions, such as thought insertion or made emotions in which we see a denial of agency over mental actions (Mellor 1970: 22).

One thing we wish to explain about this symptom is why such patients deny that they are the agents of actions which often appear to be perfectly normal, controlled, actions. A promising approach has been an attempt to understand this as a disorder of consciousness. Specifically it is proposed that such patients lack (or have a deficient) sense of agency (Frith 1979; Frith et al. 2000). Whereas in healthy individuals our actions are typically accompanied 
by a representation of our own agency which at least sometimes becomes a conscious sense, serving the purpose of allowing us to distinguish the effects of our actions on the world from the effects of other people or events, it is proposed that the mechanisms for eliciting this sense are deficient in those suffering from delusions of control. There are some actions for which no sense of agency is experienced, ultimately these actions are attributed to some other source.

So what is this sense of agency? There are a variety of confusing and jargonistic characterisations of this experience (for example, Polito et al. 2013, derive a questionnaire from 24 different terms used to describe feelings of agency). The most important features of these characterisations tend to converge on the emphasis of certain features of the experience. It is an experience of oneself as the agent of action; that is it is about oneself as the self who is in control of action. It is above all a feeling, typically taken to be ubiquitous but not often the focus of attention (Gallagher 2000; Synofzik et al. 2008). As such, although it is related to declarative judgements about agency, it can also exist independently of such judgements.

As one of the better understood self-experiences the sense of agency has become a popular case study in consciousness research, though still not as popular as colour vision. As such, theories about this experience have been formed independently of the study of psychopathology. However, if such theories are to be successful, then they ought to be applicable to the psychopathological findings. Here I examine the prospects of a theory of the sense of agency or "authorship emotion" (Wegner et al. 2004: 840; Wegner 2002: 326) developed within the study of social psychology to explain the sense of agency deficit observed in delusions of control.

\section{Wegner and Colleagues' Explanation of the Sense of Agency}

Across a number of texts Wegner and colleagues develop a theory of what they call the "authorship emotion" (Wegner 2002: 326; Wegner et al. 2004: 840 ) or what I have been calling the sense of agency. They explain the sense of agency in terms of the subject making judgements of "apparent mental state causation". In essence, whenever one infers that one or other of one's mental states is the cause of an action one performs then a sense of agency is elicited for that action.

Before moving onto how such inferences are made we should note that the theory of apparent mental state causation has a number of explanatory targets. This includes the purported "illusion" of conscious will and the implications this ought to have for debate around the existence of free will. Now this is not something I will consider here-largely because it is framed in terms of an impoverished understanding of free will inherited from Libet's 
(Libet 1999; Libet et al. 1983) dualism. The discussion is impoverished as it considers only substance dualistic notions of free will and identifies materialism with hard determinism. The consequence being that no materialist notions of free will are considered options, thereby making the debate irrelevant to those who have already rejected dualism. Wegner and colleagues' theory of the sense of agency stands independent of the conscious will and free will issues.

In Wegner and colleagues' model the sense of agency is elicited when one infers that one or other of one's mental states caused the action of one's body (Wegner 2003: 67; Wegner \& Wheatley 1999: 480). If correct, then we can provide a proximal etiological explanation of the sense of agency by explaining how this inference is made. My focus here will be on how the inference may go wrong in delusions of control. Before getting there let us consider what one needs to represent in order to successfully make this inference. To do so, one must be able to represent one's mental states qua potential causes of action, represent which body in the world is one's own (i.e. have a sense of embodiment) and represent the action which is occurring or has occurred.

Next, one must represent the relationship between one or other mental state and the action of one's body as causal. This is the role of the inference to apparent mental state causation. According to Wegner and colleagues, this inference is made when 3 facts about the relationship between the mental state and action are recognised. First, the mental state must be consistent with the action in that it specifies the action that actually occurs. Second, the mental state must seem to occur at an appropriate time before the action occurs, for example a memory of an action won't be inferred as a cause of the action. Third, the thought must appear to be the only possible cause of the action, i.e. if something else, another person or gust of wind, say, could have caused the action then the inference will not be made, or at least not made with a high degree of certainty. Wegner and colleagues call these the principles of 'consistency', 'priority' and 'exclusivity' respectively (Wegner 2003: 67; Wegner \& Wheatley 1999: 482-487).

Elsewhere (Carruthers 2010: 342-350) I have reviewed the evidence for such an account and, although I ultimately reject it, I concluded that it provides a plausible explanation of alterations to the sense of agency in healthy subjects. That is, that the subject's representation of the consistency, priority and exclusivity of a mental state as the cause of an action could be important factors in eliciting the sense of agency. I take it as relatively uncontroversial that the model can explain the experiments it is designed to explain (most importantly Aarts et al. 2005; Wegner et al. 2004; Wegner \& Wheatley 1999), so I will not repeat these arguments here. Instead, I now turn to the possibility of 
this account explaining deficits in the sense of agency associated with delusions of control.

In this model, how might the deficit in the sense of agency observed in delusions of control be explained? The explanation afforded by this model is that the pathology somehow prevents the inference to apparent mental state causation being made, say by preventing the formation of one or other of the required representations or preventing their processing.

So what representation might be missing or not be available for use in the inference to apparent mental state causation? It is unlikely to be a representation of the action being performed, e.g. formed through proprioceptive and visual feedback. Whilst the absence of such a representation would prevent the appropriate inference from being made, those suffering from delusions of alien control do not seem to have any problems in forming such representations. Although there are important problems with action monitoring which we will consider below, such patients seem able to represent which action is being performed, as evidenced by the fact that in the above reports they are attributing specific actions ("my fingers pick up the pen") to another. Furthermore, those who do have difficulties in representing what action is being performed, for example deafferented patients who are prevented from seeing their bodily movements, show consistent deficits in skilled action control (Cole \& Paillard 1995; Cole et al. 2000). This is rather different from those showing delusions of control. Whilst this delusion does correlate with some motor impairments associated with catatonia (Mellor 1970: 20-22), a striking feature of delusions of control is that they seem to occur for actions which appear to be skilfully controlled (Spence 2002).

There is experimental evidence that patients suffering from delusions of control may have difficulties with the principle of priority. The principle of priority could fail to be met if the intention to perform an action seems to occur to the patient too early or too late. This could be due to an abnormal representation of the time the intention or the action seems to occur. Studies into intentional binding suggest this may well be the case.

'Intentional binding' refers to the apparent binding together in time of actions and their effects. To see this effect, subjects are first taught that pushing a button produces a certain tone. After learning this relation, subjects are asked, for subsequent trials, to estimate using a fast rotating clock face when they pushed the button and when the sound occurred. Typically for those actions which seem to be voluntary (i.e. for which the subject experiences a sense of agency) subjects estimate that the button press and tone occur closer together in time than when a movement is involuntary, say a twitch caused by TMS (Haggard et al. 2002). 
This binding is thought to be related to the subject predicting that the tone will sound and to the subject actually hearing the tone. In a condition where the tone frequently followed the button press ( $75 \%$ of the time), and thereby typically occurred as expected, subjects reported their button press as occurring later in time than baseline (where the tone never followed the button press) even in those instances where the tone did not sound. This did not occur in runs where the tone randomly followed the button press (50\% of trials) and was thereby not predictable. As such, it seems that binding may be tightly linked to the subject predicting the effect of their action. Furthermore, in the later condition, in those trials where the tone did, in fact, sound, the binding effect was again observed (Moore \& Haggard 2008).

Unfortunately, there is no data on binding which has tested specifically for a relationship with delusions of alien control. However, some suggestive results come from Voss and colleagues' (2010) examination of intentional binding in those diagnosed with paranoid schizophrenia. In this study subjects were asked only to judge the time at which they pushed the button and not the time of the tone. In the experimental conditions the tone occurred $0.250 \mathrm{~s}$ after the button press either $75 \%$ or $50 \%$ of the time (Voss et al. 2010: 3106). The remaining time, no tone was produced. Timing judgements on these trials were compared to timing judgements in a baseline condition in which button presses where never followed by a tone (Voss et al. 2010: 3106). Timing judgements in the baseline trials were not significantly different between patients and healthy controls, suggesting that patients were not impaired in making timing judgements per se.

In both trial conditions and for both groups the perceived time of the button press was later than baseline (Voss et al. 2010: 3108). This effect was significantly larger for patients than controls (Voss et al. 2010: 3108). In addition to this difference, the pattern with which binding manifested differed between groups. As previously found, button presses were perceived as occurring later by healthy controls in the $75 \%$ condition regardless of whether or not the tone actually sounded (Voss et al. 2010: 3108). In contrast, binding was only observed for patients in the $75 \%$ condition when the tone actually sounded (Voss et al. 2010: 3108). This suggests that the predictability of the tone does not influence binding for patients. Furthermore, this effect was positively correlated with positive symptoms (Voss et al. 2010: 3110). As the delusion of alien control is one of (many) positive symptoms, this opens the door for the possibility that this effect is related to delusions of alien control.

If we take intentional binding as a straight forward index of the presence of the sense of agency, then we may be tempted to interpret these findings as showing that those suffering from paranoid schizophrenia experience a greater sense of agency over the tone than controls. After all, the intentional binding effect is greater in this group. Such a reading could be a problem for 
the claim that the sense of agency is deficient in delusions of alien control, provided further studies show that greater binding is related to delusions of alien control specifically and not a diagnosis of paranoid schizophrenia. If this is not shown, then this is not a problem at all, as only delusions of alien control and not the syndrome of paranoid schizophrenia are thought to involve a deficit in the sense of agency.

Further, binding seems to be occurring for different reasons in patients than in controls. In particular, in healthy controls binding is closely related to the subject's prediction that the tone will sound. In contrast, in patients binding does not seem to be related to prediction of the tone. Additionally, whilst binding is related to the actual hearing of the tone in healthy controls, this relationship is much stronger in patients. Whilst we may be reasonably confident that binding correlates with the presence of the sense of agency in healthy controls, it is not straightforward to infer that patients experience a stronger sense of agency because of a greater degree of binding in patients. A breakdown in whatever mechanism is in common between the sense of agency and intentional binding (possibly motor prediction) needn't have the same effect on both. It may increase one and decrease the other. The mere strengthening of intentional binding in patients does not, on its own, challenge the claim that there is a sense of agency deficit associated with delusions of alien control.

These studies suggest a reason why patients may be unable to make the inference to apparent mental state causation for some actions. At least when there are pronounced auditory consequences of action, the binding data suggests that patients may represent their action as occurring later than normal. This may lead to a longer-than-expected delay between their intention to act (or whatever mental state is a potential cause of the action) and the action itself. As such, the principle of priority may be violated. To the patient, the action may seem to occur too late to be plausibly caused by the intention to act. As such, the inference to apparent mental state causation is not made and the sense of agency is not elicited, or at least is not as strong.

This is a vague formulation as it stands, but I think it is good for Wegner and colleagues so far. Even without entering into specifics as to how the principle of priority works, we have a hypothesis that can be tested against the already existent data. In particular an advocate of Wegner and colleagues' model may think that we have good reason to put forward the following hypothesis: The sense of agency is not elicited for some actions of those suffering from delusions of alien control, as the inference to apparent mental state causation is not made due to a violation of the principle of priority. At base, the problem in this symptom is that the patient's actions seem to the patient to occur too late. Is there any independent evidence in support of this hypothesis? 


\section{Self-Tickling}

There is some reason to think that delusions of alien control are associated with specific abnormalities in tactile experience. Here I will suggest that Wegner and colleagues' model is consistent with these abnormalities, as the timing problems identified above (which would violate the principle of priority) may also explain these findings. Blakemore and colleagues (2000) compared tactile sensations in those who had experienced delusions of control and verbal hallucinations within 6 weeks of testing (diagnosed with schizophrenia, bipolar disorder or depression) to those with these diagnosis but not verbal hallucinations or delusions of alien control and healthy control subjects (Blakemore et al. 2000: 1134). Subjects were asked to rate the apparent intensity, painfulness, tickliness, pleasantness and irritatingness of the touch of a foam cube on their palm. In one condition, the cube was moved by an experimenter using a lever, in another the subject themselves moved the foam (Blakemore et al. 2000: 1134). Healthy controls and those suffering from schizophrenia, bipolar disorder or depression but not experiencing verbal hallucinations or delusions of control rated the tickliness, intensity and pleasantness of self-produced touch significantly lower than the touch produced by another person (Blakemore et al. 2000: 1136). However, those who had recently experienced delusions of control or verbal hallucinations reported no such difference (Blakemore et al. 2000: 1136).

Traditionally, this finding has been interpreted as evidence for the comparator model account of the sense of agency. It is thought that a problem with predicting the tactile outcomes of actions is a common cause of both the failure to attenuate self-produced tactile sensation and the deficit in the sense of agency. An auxiliary hypothesis would be that a prediction of tactile stimuli attenuates the felt strength of actual tactile stimuli when they, in some sense, match. This is so despite the fact that the relationship between deficits in the sense of agency over bodily action and the lack of attenuation is not entirely clear in this study. The group showing a lack of attenuation of self-produced tactile stimuli was composed of 17 patients (some diagnosed with schizophrenia, others with one of the affective disorders), all of whom had recently experienced verbal hallucinations. In contrast, only 6 of the 17 had recently experienced delusions of alien control (Blakemore et al. 2000: 1134). In the comparator model, it is not clear why those suffering from verbal hallucinations should share a common deficit with those suffering from deficits in the sense of agency over their bodily actions. In Wegner and colleagues' model, in contrast, deficits in the sense of agency over mental actions could arise from the same (or the same kind of) problem as deficits in the sense of agency over bodily actions, namely, a problem in inferring that one's current thoughts (or verbal imagery) are caused by one's previous thoughts. 
My question here is: can Wegner and colleagues' model explain this finding? Is there any reason on Wegner and colleagues' account that sense of agency deficits ought to be associated with a failure to attenuate self-produced tactile stimuli? Wegner and colleagues' model stands up to this finding surprisingly well, given the minor role sensory feedback plays in this model. Above I suggested that there is some reason to think that the patient suffering from delusions of alien control perceives their actions as occurring too late. Such a misrepresentation of the time of the action resulting in the tactile feedback could, potentially, result in both abnormalities. As above, the misrepresentation of the time of the action may prevent the principle of priority from being met and, thereby, prevent the inference to apparent mental state causation from occurring. In such a case, the sense of agency would not be elicited (or, at least, it would be significantly weakened). Similarly, if the tactile feedback seems to occur later than predicted, perhaps this could violate a represented match between them, thereby preventing the prediction from being used to attenuate the actual feedback.

Although these findings are not yet explained, we have seen so far that there is data which is consistent with a Wegner-and-colleagues' type of account of the sense of agency deficits observed in delusions of alien control. There is some reason to suppose that a problem in the represented time of action prevents the sense of agency from being elicited due to consistent violation of the principle of priority. This hypothesis has potential explanatory force beyond the data which motivates it. That being said, I argue next that ultimately this kind of account will be unsatisfactory as it makes a false prediction, namely that there will be a theory of mind deficit associated with delusions of control.

\section{Action Monitoring}

There are a variety of studies which suggest that those suffering from delusions of control have a degraded awareness of their own actions (see Carruthers 2012 for review). For example, patients suffering from delusions of control make more errors and take longer to correct them in simple perceptual discrimination tasks (Stirling et al. 1998: 679) and worse at recognising drawings they themselves had previously made (Stirling et al. 1998: 680) than pathological and healthy controls. Such patients are also worse at identifying whether or not they or someone else are performing an action they see displayed on a screen when they were performing the same action as displayed (Daprati et al. 1997: 79) and fail to notice distortions given to the visual feedback of their actions of up to 40 degrees (Franck et al. 2001), whereas healthy subjects are sensitive to distortions of around 15 degrees (Farrer et al. 2003). 
Here I will focus on one study from Frith and Done (1989) as an example of these action monitoring deficits which are problematic for Wegner and colleagues' model. In this study, subjects played a video game in which they fired a gun at a target which appeared on the left or right of a screen by moving a joystick left or right. Errors were induced by altering the relationship between joystick movements and the direction the gun fired. In some trials, a rightward movement fired the gun right, in others a rightward movement fired the gun left (and vice versa). Although no worse than healthy controls or those diagnosed with schizophrenia but not suffering from delusions of control at keeping track of this relationship (Frith \& Done 1989: 362), those suffering from delusions of control failed to correct errors when they couldn't see the direction the bullet moved after being fired. Until they have seen the trajectory, they do not know which way they fired.

Wegner and colleagues' model cannot easily accommodate such findings. First, the apparent delay in action, so powerful above, seems unlikely to be helpful here. Whilst intuitively we may expect a delay in error correction in those suffering from delusions of control, we would expect this to be of a similar size to the delay suggested by the Voss and colleagues' study. In this study those suffering from schizophrenia experienced their actions as occurring in the order of 10s of milliseconds later than controls (Voss et al. 2010: 3107). Similarly, Blakemore and colleagues found that by using a machine to induce a 200ms delay between action and stimulus that subjects could experience self-produced stimuli as as tickly as other-produce stimuli (Blakemore et al. 1999). In contrast, in Frith and Done's (1989) study, the time subjects had to correct their actions before the bullet became visible was up to two orders of magnitude greater at 2s. Intuitively, it seems those suffering from delusions of control would be able to correct within this time if their only problem were the misrepresentation of the time of their action. Although this is merely suggestive evidence, it is clear that some positive evidence would be needed to show why timing delays (underlying the violation of the principle of priority) could explain self-monitoring deficits.

Perhaps, then, an advocate of a Wegner-and-colleagues' type of model could resort to a more orthodox explanation of this finding. Perhaps the patient lacks some representation of their action which prevents error correction in the absence of visual feedback and prevents the sense of agency from being elicited. There seem to be two candidate representations here. A deficit in representing the action itself, say by sensory feedback, or in the intention to perform the action may be deficient. If there is a deficiency in sensory feedback specifying the action performed, the patient would be unable to correct errors as they do not know what they have done. Similarly, they would not be able to make the inference to apparent mental state causation and thereby they would have no sense of agency. If, on the other hand, the deficit is in their representation of their intentions, then perhaps they cannot rapidly 
correct errors as they do not know what they were trying to do. Similarly, they would be unable to infer their intention caused their action and so no sense of agency would be elicited.

There is reason to suppose that non-visual sensory feedback is reasonably intact for those suffering from delusions of alien control. They can discriminate the actions which they only feel themselves perform from those which they see. For example, in Daprati and colleagues (1997) patients suffering from delusions or hallucinations (as a group) were able to judge that they were being shown a video of someone else acting when the seen action differed from what they felt themselves doing. At least it seems there is not a complete failure of sensory feedback. As such, a more plausible hypothesis is that these patients have difficulties in knowing their own intentions.

However, there is no reason to suppose that those suffering from delusions of control have difficulty knowing their own intentions. There is considerable evidence that there is a theory of mind deficit in schizophrenia (see Brune 2005; Harrington et al. 2005 for reviews). This seems to offer some support for claim that those suffering from delusions of control have such a deficit. However, a closer examination of which patients suffer from a theory of mind deficit is not so positive. It seems that those with negative signs (such as psychomotor poverty) and positive signs (disorganised speech) suffer the greatest deficits, with ambiguous findings for those with paranoia and no deficit that is specific to the first rank symptoms, which includes delusion of alien control (Corcoran et al. 1995; Greig et al. 2004; Mazza et al. 2001; Pickup and Frith 2001). As such, the presence of theory of mind deficits associated with the syndrome of schizophrenia does not constitute evidence that those suffering from delusions of control have difficulty in knowing their own intentions. Here I describe the evidence that suggests that there is no theory of mind deficit displayed by those with such symptoms.

Corcoran and colleagues (1995) tested schizophrenic patients' abilities to infer the intentions of others from indirect speech. Subjects were told a series of stories about two characters, each of which ended with one character hinting that they wanted the other character to do something for them (Corcoran et al. 1995: 12). Overall the performance of schizophrenic patients on this test was significantly worse than both healthy controls and patients with anxiety or depression (Corcoran et al. 1995: 8-9). However, the worst performance in this test came from those with negative and positive behavioural signs (although only one patient was in the positive sign group). Paranoid patients also did significantly worse than controls (Corcoran et al. 1995: 10). In contrast, those with only passivity experiences, including delusions of alien control, were as good at this task as either control group or schizophrenic patients currently in remission (Corcoran et al. 1995: 10). This suggests that there is a symptom- 
specific deficit in theory of mind in schizophrenia; however, it is not related to delusions of alien control.

Similar findings have been obtained for first- and second-order false belief tests. Second-order tasks test for the subject's ability to attribute to someone a false belief about someone else's belief. An example of this kind of test involves 3 characters: Stephen, Ann and a nurse. Stephen and Ann are watching TV in the lounge room of a hospital ward, where they are patients. Ann goes for a walk, leaving Stephen alone in the lounge watching TV. A nurse comes in and tells Stephen that the TV is going to be moved to the dining room downstairs. Stephen stays in the lounge and the nurse takes the TV down to the dining room. On the way she passes Ann and tells her that the TV is to be put in the dining room. Both Stephen and Ann know that the TV has been moved but neither know that the other has been told this. An hour later Stephen is looking for Ann, another patient tells him that Ann said she was going to watch TV. Stephen hurries off to find Ann. The test question is "where will Stephen look for Ann?” (Pickup and Frith 2001: 219). The correct answer is in the lounge. In order to answer correctly subjects must attribute to Stephen the false belief that Ann still believes the TV is in the lounge.

Pickup and Frith (2001) compared the performance of schizophrenic patients to healthy controls and psychiatric controls on these tests. Schizophrenic patients were divided into four groups by symptoms. These were i) those with positive or negative behavioural signs ii) paranoia but no behavioural signs iii) passivity symptoms but no behavioural signs or paranoia iv) remission (i.e. none of the above symptoms). Only one subject fell into group iii) passivity, which makes interpreting these data difficult, to say the least. However, the results are suggestive and, given the consistency with the above findings, worthy of consideration. It was found that all schizophrenic patients did as well as both control groups on the first-order false belief tests (Pickup \& Frith 2001: 212). Only those with behavioural signs were significantly worse than the control groups on second-order tests. There was an insignificant trend for paranoid patients to be worse than controls (Pickup \&Frith 2001: 213). The one patient experiencing passivity delusions performed as well as controls on both tests (Pickup \& Frith 2001: 214). Although it is difficult to draw lessons from a single case, it does seem that we have some grounds for thinking that theory of mind deficits in schizophrenia are specific to the behavioural signs groups.

Mazza and colleagues (2001) have found a similar pattern of results using different comparison groups. This study compared three groups i) psychomotor poverty ii) disorganised speech and iii) reality distortion (Mazza et al. 2001: 301-302). The reality distortion group contained those with passivity experiences and paranoid delusions. Groups i and ii correspond to negative and positive behavioural signs. For my purposes here, the interesting finding is that the reality distortion group did well on first order false belief tests, being $75 \%$ 
and $87 \%$ correct on the two tests administered. A similar pattern was found for second order tests where again $75 \%$ and $87 \%$ patients were correct (Mazza et al. 2001: 303-305). This was worse than controls but still systematically correct, suggesting that failure in the test was not due to a theory of mind deficit per se. Unfortunately, the authors did not further analyse their findings by comparing those with paranoia to those with passivity experiences, so we don't know who made the errors on these tests.

Across these findings what we see is that the theory of mind deficit associated with schizophrenia is not associated with delusions of alien control per se. This is not definitive, of course. Again, the tests do not compare those with delusions of control specifically to those suffering from schizophrenia but without delusions of control. Instead, such patients are in a group with those suffering from other passivity experiences, such as verbal hallucinations or delusions of thought insertion, or else grouped with those suffering from paranoid delusions. Furthermore, they are not direct tests of the patients knowledge of their own intentions, but, rather, inferences about their general capacity for theory of mind is being inferred from tests of their knowledge of others communicative intentions or false beliefs about beliefs. It is possible, although unlikely, that patient's knowledge of their own intentions is deficient, whilst the rest of their theory of mind remains intact. These cautionary notes aside, we definitely lack reason to suppose that patients suffering from delusions of control have deficient knowledge of their own intentions. Ultimately, then, the hypothesis that those suffering from delusions of control lack knowledge of their own intentions, which would render a Wegner-andcolleagues' type model consistent with the difficulties in action monitoring observed here, is unsupported. If this hypothesis predicts a general theory of mind deficit associated with delusions of control, then it has already been falsified.

Wegner and colleagues' model thereby seems to have difficulty accommodating action monitoring deficits associated with delusions of alien control. In order to maintain this model, an advocate needs to break the link between sense of agency deficits and action monitoring deficits seen in delusions of alien control. It needs to be shown that, despite both being core to delusion of alien control, there is no necessary link between sense of agency deficits and action monitoring deficits of this kind. However, given that we already have an explanation, namely the comparator model, which explains why both deficits should occur together in delusions of alien control the task to split this link is a daunting one. Whilst we should acknowledge the possibility of a Wegner-and-colleagues' type explanation of the delusion of alien control, especially given the successes discussed here, at this stage it seems to be an implausible explanation which would require the link between the sense of agency and action monitoring to be fundamen-tally reconceptualised. 


\section{Conclusion}

Here I have examined the possibility of Wegner and colleagues' explanation of the sense of agency over bodily actions being extended to account for sense of agency deficits associated with delusions of alien control. Data from intentional binding studies suggests that such patients may represent their actions as occurring later than they should. If this is enough to violate the principle of priority, then this is consistent with Wegner and colleagues' account of the sense of agency. As well as explaining intentional binding and sense of agency deficits, the hypothesis that these patients represent their actions as occurring too late may also explain why these patients do not show sensory attenuation for self-produced stimuli. Despite these successes, Wegner and colleagues' model seems to face great difficulty accommodating action monitoring deficits observed in those suffering from delusions of control. Whilst there are hypotheses which, if true, would link sense of agency deficits to action monitoring deficits, these hypotheses turn out to be false or at best unsupported. The model might be true even given these findings, but this would require an implausible reconceptualization of the link between the sense of agency and action monitoring. Ultimately, then, Wegner and colleagues' model is unlikely to be true.

Aarts, H., Custers, R., and Wegner, D. M. 2005. On the inference of personal authorship: enhancing experienced agency by priming effect information. Consciousness and Cognition, 14: 439-458.

Blakemore, S., Frith, C., and Wolpert, D. 1999. Spatio-temporal prediction modulates the perception of self-produced stimuli. Journal of Cognitive Neuroscience, 11: 551559.

Blakemore, S.-J., Smith J., Steel, R., Johnstone, E. C., and Frith, C. D. 2000. The perception of self-produced sensory stimuli in patients with auditory hallucinations and passivity experiences: evidence for a break-down in self monitoring. Psychological Medicine, 30: 1131-1139.

Brune, M. 2005. "Theory of mind" in schizophrenia: a review of the literature. Schizophrenia Bulletin, 31(1): 21-42.

Carruthers, G. 2010. A problem for Wegner and colleagues' model of the sense of agency. Phenomenology and the Cognitive Sciences, 9: 341-357.

Carruthers, G. 2012. The case for the comparator model as an explanation of the sense of agency and its breakdowns. Consciousness and Cognition, 21(1): 30-45.

Cole, J., and Paillard, J. 1995. Living without touch and peripheral information about body position and movement: Studies with deafferented subjects. Bermudez, J. L., Marcel, A., Eilan, N. The Body and the Self. Cambridge: MIT Press.

Cole, J., Sacks, O., and Waterman, I. 2000. On the immunity principle: a view from a robot. Trends in Cognitive Science, 4 (5). 
Corcoran, R., Mercer, G., and Frith, C. D. 1995. Schizophrenia, symptomatology and social inference: investigating "theory of mind" in people with schizophrenia. Schizophrenia Research 17: 5-13.

Daprati, E., Franck, N., Georgieff, N., Proust J., Pacherie, E., Dalery, J., and Jeannerod, M. 1997. Looking for the agent: an investigation into consciousness of action and self-consciousness in schizophrenic patients. Cognition 65: 71-86.

Farrer, C., Franck, N., Paillard, J., and Jeannerod, M. 2003. The role of proprioception in action recognition. Consciousness and Cognition, 12(4), 609-619.

Franck, N., Farrer, C., Georgieff, N., Marie-Cardine, M., Dalery, J., d' Amato, T., and Jeannerod, M. 2001. Defective Recognition of One's Own Actions in Patients with Schizophrenia. American Journal of Psychiatry, 158(3), 454-459.

Frith, C.D. 1979. Consciousness, Information Processing and Schizophrenia. British Journal of Psychiatry, 134, 225-235.

Frith, C. D., and Done, D. J. 1989. Experiences of Alien Control in Schizophrenia Reflect a Disorder in the Central Monitoring of Action. Psychological Medicine, 19: 359363.

Frith, C. D., Blakemore, S.-J., and Wolpert, D. M. 2000. Abnormalities in the Awareness and Control of Action. Philosophical Transactions of the Royal Society of London Series B-Biological Sciences, 355, 1771-1788.

Gallagher, S. 2000. Philosophical Conceptions of the Self: Implications for Cognitive Science. Trends in Cognitive Science, 4(1): 14-21.

Graham, G., and Stephens, G. L. 1994. Mind and Mine. Graham, G., and Stephens, G. L., eds., Philosophical Psychopathology, Cambridge: MIT Press: 91-110.

Greig, T. C., Bryson, G. J., and Bell M. D. 2004. Theory of mind performance in schizophrenia: diagnostic, symptom and neuropsychological correlates. The Journal of Nervous and Mental Disease, 192(1).

Haggard, P., Clark, S., and Kalogeras, J. 2002. Voluntary action and conscious awareness. Nature Neuroscience, 5(4).

Harrington, L., Siegert, R. J., and McClure, J. 2005. Theory of mind in schizophrenia: a critical review. Cognitive Neuropsychiatry, 10(4): 249-286.

Libet, B. 1999. Do we have free will? Journal of Consciousness Studies, 6(8-9): 47-57.

Libet, B., Gleason, C. A., Wright, E. W., and Pearl, D. K. 1983. Time of Conscious Intention to Act in Relation to Onset of Cerebral Activity Readiness Potential: the Unconscious Initiation of a Freely Voluntary Act. Brain, 106: 623-642.

Mazza, M., De Risio, A., Surian, L., Roncone, R. and Casacchia, M. 2001. Selective impairments of theory of mind in people with schizophrenia. Schizophrenia Research, 47: 299-308.

Mellor, C. S. 1970. First Rank Symptoms of Schizophrenia. British Journal of Psychiatry, 117: $15-23$. 
Moore, J., and Haggard, P. 2008. Awareness of action: Inference and prediction. Consciousness and Cognition, 17(1): 136-144.

Pickup, G., and Frith, C. D. 2001. Theory of mind impairments in schizophrenia: symptomatology, severity and specificity. Psychological Medicine, 31: 207-220.

Polito, V., Barnier, A. J., and Woody, E. Z. 2013. Developing the Sense of Agency Rating Scale SOARS: An empirical measure of agency disruption in hypnosis. Consciousness and Cognition, 22(3), 684-696. doi:10.1016/j.concog.2013.04.003

Spence, S. A. 2002. Alien motor phenomena: a window on to agency. Cognitive Neuropsychiatry, 7(3): 211-220.

Stephens, G. L., and Graham, G. 2000. When Self-Consciousness Breaks: Alien Voices and Inserted Thoughts, Camridge: MIT Press.

Stirling, J., Hellewell, J., and Quraishi, N. 1998. Self-monitoring dysfunction and the schizophrenic symptoms of alien control. Psychological Medicine, 28: 675-683.

Synofzik, M., Vosgerau, G., and Newen, A. 2008. Beyond the Comparator Model: A Multifactorial two-step account of agency. Consciousness and Cognition, 17: 219239.

Voss, M., Moore, J., Hauser, M., Gallinat, J., Heinz, A., Haggard, P. 2010 Altered awareness of action in schizophrenia: a specific deficit in predicting action consequences. Brain, 133: 3104-3112.

Wegner, D. M. 2002. The Illusion of Conscious Will, Cambridge: MIT Press.

Wegner, D. M. 2003. The mind's best trick: how we experience conscious will. Trends in cognitive sciences, 7(2).

Wegner, D. M., Sparrow, B., and Winerman, L. 2004. Vicarious Agency: Experiencing control over the movements of others. Journal of Personality and Social Psychology, 86(6): 838-848.

Wegner, D. M., and Wheatley, T. 1999. Apparent mental causation: sources of the experience of will. American Psychologist, 54(7): 480-492. 